\title{
DO ANTIBIOTIC INTRAMEDULLARY DOWELS ASSIST IN ERADICATING INFECTION IN TWO-STAGE RESECTION FOR SEPTIC TOTAL KNEE ARTHROPLASTY?
}

\author{
Matthew R. Zielinski MS ${ }^{1}$ \\ Mary Ziemba-Davis BA ${ }^{2}$ \\ Lucian C. Warth MD ${ }^{2,3}$ \\ Brian J. Keyes DO ${ }^{2}$ \\ R. Michael Meneghini MD ${ }^{2,3}$ \\ ${ }^{1}$ Indiana University School of Medicine, Indianapolis, IN \\ ${ }^{2}$ Indiana University Health Orthopedics, Fishers, IN \\ ${ }^{3}$ Indiana University School of Medicine, Department of Orthopaedic Surgery, Indianapolis, IN
}

R. Michael Meneghini, MD (corresponding author)

Indiana University School of Medicine, Department of Orthopaedic Surgery

Indiana University Health Orthopedics, 13000 East 136th Street, Suite 2000, Fishers, IN 46037

Phone: 317-688-5980; Fax: 317-678-3222

Email: rmeneghi@iuhealth.org

This is the author's manuscript of the article published in final edited form as:

Zielinski, M. R., Ziemba-Davis, M., Warth, L. C., Keyes, B. J., \& Meneghini, R. M. (2019). Do Antibiotic Intramedullary Dowels Assist in Eradicating Infection in Two-Stage Resection for Septic Total Knee Arthroplasty? The Journal of Arthroplasty. https://doi.org/10.1016/j.arth.2019.05.022 


\section{DO ANTIBIOTIC INTRAMEDULLARY DOWELS ASSIST IN ERADICATING INFECTION IN TWO-STAGE RESECTION FOR SEPTIC TOTAL KNEE ARTHROPLASTY?}

\section{Abstract}

Introduction: Evidence suggests approximately $40 \%$ of intramedullary (IM) canals are culture positive at resection for infected knee arthroplasty. While commonly utilized, no clinical data on the efficacy of antibiotic-eluding IM dowels exists. We quantified treatment success with and without the use of antibiotic-eluding IM dowels in two-stage treatment of periprosthetic knee infection using static and articulating antibiotic cement spacers.

Methods: 109 consecutive patients who underwent two-stage treatment for periprosthetic knee infection were reviewed. Treatment failure, defined as repeat resection before reimplantation or recurrent infection within 6 months of reimplantation, was evaluated based on spacer type and use of IM dowels, accounting for infection type, and systemic host and local extremity grade.

Results: After exclusions for confounds, articulating spacers without IM dowels were used in 49 $(57.7 \%)$ cases, articulating spacers with IM dowels in 14 cases $(16.5 \%)$, and static spacers with IM dowels in $22(25.9 \%)$ cases. Treatment success regardless of infection classification was $85.7 \%$ for articulating spacers with IM dowels, $89.8 \%$ for articulating spacers without IM dowels, and $68.2 \%$ for static spacers with IM dowels $(p=0.074)$. In chronically infected poor hosts with compromised extremities, treatment success remained highest in patients with articulating spacers with $(90.9 \%)$ or without $(92.9 \%)$ IM dowels compared to static spacers with IM dowels $(68.4 \%)(p=0.061)$.

Conclusion: Findings suggest that the use of IM dowels did not enhance infection eradication above and beyond that observed for articulating spacers alone, including in the worst cases involving chronically infected poor hosts with compromised extremities. 
27 Keywords: periprosthetic joint infection; knee infection; knee revision; intramedullary dowel;

28 antibiotic spacer; two-stage treatment

29

30 


\section{Introduction}

Two-stage treatment of periprosthetic knee infection employs either articulating or static high dose antibiotic-eluding tibiofemoral cement spacers between resection and reimplantation, with or without antibiotic-eluding intramedullary (IM) dowels. Systematic reviews comparing articulating and static spacers do not address IM dowels but do provide general consensus that the two spacer types equivalently eradicate infection although articulating designs result in improved range of motion and function following reimplantation. [1-3] However, one review observed a significantly lower reinfection rate with articulating spacers, [4] and all four reviews commented on the absence of comprehensive and high quality comparative studies. After review and consideration of the available evidence, the 2018 International Consensus Meeting on Periprosthetic Joint Infection recommended the use of articulating spacers unless static spacers are indicated by severe bone loss, ligament insufficiency, or soft tissue compromise; [5] however, the International Consensus group did not comment on the use of IM dowels to enhance treatment and facilitate the eradication of infection.

Although studies on spacer type and efficacy are limited, data on the need for and efficacy of antibiotic-eluding IM dowels are non-existent. High-dose antibiotic IM dowels are not uncommonly used in practice to enhance antibiotic delivery specifically into the IM tibial and femoral canals. Hanssen and Spangehl [6] recommended antibiotic cement IM dowels based on the fact that "In roughly $1 / 3$ of infected knee replacements without stems there is an extension of the infectious process into the medullary canals of the femur or tibia." Their recommendation was based on the 1997 study by McPherson and colleagues [7] who reported that $39 \%$ of 71 positive growth cultures taken at resection were from medullary canal specimens. Yet, despite this single study over two decades ago supporting the rationale for antibiotic cement IM dowels 
being commonly used in practice, studies on the efficacy of and justifying the use of antibioticeluding IM dowels do not currently exist. The purpose of this study was to quantify treatment success rates with and without the use of antibiotic-eluding IM dowels in two-stage treatment of periprosthetic knee infection using both high-dose antibiotic cement static and articulating spacers.

\section{Methods}

\section{Study Sample}

One-hundred and nine consecutive patients who underwent two-stage treatment for periprosthetic knee infection by one of four academic surgeons between 08/30/2010 and 07/20/2017 were retrospectively reviewed with Institutional Review Board approval. Surgeries were performed with the same perioperative protocols at one of two tertiary referral centers for patients with more severe medical comorbidities. Twenty-four cases were excluded because they involved extensor mechanism repair (9), resection of an existing antibiotic spacer (6), skin reconstruction (4), resection of a distal femoral replacement (3), and resection of $11.5 \mathrm{~cm}$ of necrotic distal femoral bone (1). One additional single case in which a static spacer without dowels was used also was excluded.

\section{Surgical Procedures}

During initial stage resection, procedures were typically performed under tourniquet until exposure and debridement was completed. Then prior to cementation of the antibiotic cement spacer, the tourniquet was let down to obtain hemostasis and also to prevent excessive cement interdigitation to facilitate spacer removal at second stage reimplantation. Procedures were typically performed through an anterior longitudinal incision, ellipsing and excising any tenuous regions of the previous incision to facilitate wound approximation and postoperative healing. A 
median parapatellar arthrotomy was performed in all cases and if extensile exposure was needed a quadriceps snip was performed. Five cultures were routinely obtained from within the knee joint and sent to microbiology for analysis. Meticulous and careful exposure was performed with synovial tissue excision and debridement of the suprapatellar pouch, and medial and lateral gutters, and the retropatellar fat pad/scar tissue was excised to eradicate the infection and facilitate exposure.

Femoral and tibial implants were carefully removed with a combination of reciprocating saws and osteotomes. All residual cement was removed with a combination of osteotomes and rongeours, taking care to preserve as much bone as possible. Particular attention was paid to any cement in previous drill or peg holes to ensure removal and a thorough debridement. Femoral and tibial intramedullary canals were opened and debrided in all cases using reverse curettes, scrapers, and flexible reamers. A careful assessment of femoral and tibial bone loss was performed to determine whether an articulating spacer was feasible, and to determine the future reimplantation and reconstruction strategy. With the knee in flexion, the posterior capsule and infected synovium were excised and debrided carefully to avoid damaging posterior neurovascular structures. The polyethylene patella component was removed in all cases and all visible cement was removed from patellar, femoral, and tibial bone. The knee joint was then irrigated with 6 liters of bacitracin-containing pulsed lavage. The tourniquet was deflated and hemostasis was obtained. Particular attention was paid to irrigating all bone surfaces and periarticular tissues thoroughly, as well as into the femoral and tibial canals. Periarticular tissues were soaked in a dilute betadine solution of $0.35 \%$ for 3 minutes, followed by 3 more liters of normal saline pulsed irrigation. 
An articulating or static spacer was implanted as appropriate. In cases where adequate

100

101

102

103

104

105

106

107

108

109

110

111

112

113

114

115

116

117 bone stock was available and an articulating spacer was indicated $(n=63)$, one of three different types were used: 13 (20.6\%) pre-fabricated spacers (REMEDY® Knee Spacer, OsteoRemedies, Memphis, TN), 23 (36.5\% ) molded antibiotic tibial and femoral spacers (Zimmer Biomet, Warsaw, IN), and 27 (42.9\% ) molded cement femoral components and all-poly tibial inserts implanted with high-dose antibiotic cement (Depuy Synthes, Warsaw, IN). Indications for static spacers were at the discretion of the surgeon and included substantial bone loss and collateral ligament integrity. In cases where a static spacer was deemed appropriate $(n=22)$, high-dose antibiotic-laden cement was molded to the distal femur and proximal tibia. IM dowels were used with all static spacers and, depending on surgeon preference, were formed around a central reinforcing Steinmann pin (Key Surgical, Minneapolis, MN) or nail (Fixation Recon Nail, Zimmer Biomet, Warsaw, IN) or constructed without a central metal pin utilizing a cement gun nozzle (Stryker, Mahwah, NJ) lubricated with normal saline to facilitate cement removal once cured. Spacer and dowel constructs are shown in Figure 1. The distribution of femoral and tibial IM dowels is presented in Table 1 based on spacer type and method of dowel construction. Spacers and dowels utilized high-dose antibiotic cement (Biomet Cobalt; Stryker Simplex P, Zimmer Palacos) and dosing appropriate for the cement type. For Cobalt and Palacos cement, 2 grams of vancomycin and 0-2 grams tobramycin was used, and for Simplex P cement, 3-4 grams of vancomycin and 3.6 grams tobramycin were utilized. In Simplex P, one cubic centimeter of methylene blue per cement batch was added to provide color to facilitate visualization upon removal at reimplantation. In indicated cases of multi-organism and recalcitrant infections, an antifungal (50 micrograms Amphotericin B) was added to the cement. 
A hemovac drain was used in all cases and closure was performed in standard

fashion. Patients were discharged with 6 weeks of intravenous, primarily single antibiotic therapy (ampicillin, cefazolin, cefepime, ceftaroline, ceftriaxone, daptomycin, vancomycin, or zosyn). If preoperative or intraoperative cultures failed to grow an organism ("culturenegative"), patients were typically treated with dual-antibiotic broad-spectrum intravenous therapy for the 6-week interval. Upon completion of intravenous antibiotics, all patients underwent a two-week antibiotic-free interval with serologic testing to include erythrocytesedimentation rates (ESR) and C-reactive protein (CRP) counts. If either lab was elevated or clinical suspicion was high, the knee was aspirated for analysis of cell counts, differentials, and cultures prior to reimplantation.

\section{Measures}

The electronic medical record was used to compile study data: patient sex, age, body mass index (BMI) in $\mathrm{kg} / \mathrm{m}^{2}$, intraoperative culture results (infectious organisms), McPherson et al./University of Southern California Classification for Prosthetic Joint Infection [8], whether traditional Musculoskeletal Infection Society (MSIS) criteria [9] were met, spacer type, whether or not IM dowels were used and where (femur only, tibia only, or both), stage one surgical procedure operating time in minutes, and time between resection and reimplantation in months. McPherson et al.'s classification system for PJI assesses each patient according to three categories-infection type, systemic host grade (medical/immune status), and local extremity grade—resulting in a single infection classification (e.g., I-A-1; Table 2). Per McPherson et

\section{al.'s classification system, infections in chronically infected poor hosts are designated as}

IIIB and IIIC. Treatment success was defined as no repeat resection before reimplantation and no recurrent infection within 6 months of reimplantation. 


\section{Data Analysis}

Minitab 18 (State College, PA) was used for statistical analysis. Analysis of variance (F) was used to compare mean values in three groups (articulating spacers with IM dowels, articulating spacer without IM dowels, and static spacers with IM dowels). Pearson's ChiSquare $\left(\mathrm{X}^{2}\right)$ test was used to test independence among categorical variables. Post hoc $\mathrm{z}$ tests with Fishers Exact test $p$ value were used to compare specific group proportions. A significance level of $\leq 0.05$ was used for all statistical analyses.

\section{Results}

Slightly more females $(44,51.8 \%)$ than males $(41,48.2 \%)$ comprised the final sample of 85 consecutive two-stage treatment cases. Average age and BMI were 66 (SD 9.0) years and 36 (SD 9.0) kg/m², respectively. Ninety-nine percent (84/85) of sample cases met traditional MSIS criteria for PJI. The patient who did not fit traditional MSIS criteria had elevated ESR, CRP counts, and intraoperative white cell counts but the values did not meet traditional MSIS thresholds.

Articulating spacers without IM dowels were used in 49 (57.7\%) cases, articulating spacers with IM dowels were used in 14 cases (16.5\%), and static spacers with IM dowels were used in $22(25.9 \%)$ cases. Overall treatment success rates regardless of McPherson infection classification are shown for the three groups in Figure $2\left(\mathrm{X}^{2}=5.2, p=0.074\right)$. Paired comparisons indicated no statistical difference in treatment success between articulating spacers with and without IM dowels ( $85.7 \%$ vs. $89.8 \%, \mathrm{z}=0.40, p=0.646)$, and between articulating spacers with dowels and static spacers with dowels $(85.7 \%$ vs. $68.2 \%, \mathrm{z}=1.29, p=0.432)$ with the numbers available. A significantly greater treatment success rate was observed for articulating spacers without IM dowels compared to static spacers with IM dowels (89.8\% vs. 
$68.2 \%, \mathrm{z}=2.00, p=0.039)$. Treatment success rates based on the location of dowels in the femur only $(66.7 \%, 2 / 3)$, the tibia only $(0 \%, 0 / 1)$, or both the femur and the tibia $(78.1 \%, 25 / 32)$ could not be tested due to small numbers in the groups.

PJI classification is provided in Table 3 based on spacer type and the use of IM dowels with gray shading demarcating classifications associated with chronically infected poor hosts with compromised extremities (III B/C 2/3). Significantly more patients with static spacers and IM dowels (19/22, 86.4\%), followed by patients with articulating spacers and IM dowels (11/14, $78.6 \%)$, and articulating spacers without IM dowels $(28 / 49,57.1 \%)$ were chronically infected poor hosts with compromised extremities $\left(\mathrm{X}^{2}=6.807, p=0.033\right)$. As shown in Figure 2, however, among chronically infected poor hosts with compromised extremities, treatment success rates remained the highest in patients with articulating spacers with (90.9\%) and without IM dowels $(92.9 \%)$ compared to static spacers with IM dowels $\left(68.4 \%\left(\mathrm{X}^{2}=5.582, p\right.\right.$ $=0.061)$. Treatment success rates in these patients with articulating spacers with and without IM dowels $(90.9 \%$ vs. $92.9 \% ; z=0.20, p=1.000)$ did not differ in paired comparisons. There was no statistically significant difference in success rates between articulating spacers with dowels and static spacers with dowels with the numbers available (90.9\% vs. $68.4 \%, \mathrm{z}=1.64, p$ $=0.215)$. Treatment success rates were significantly higher in comparisons of articulating spacers without dowels and static spacers with dowels (92.9\% vs. 68.4\%, $\mathrm{z}=2.08, p=0.047)$. Patient sex, age, BMI, infectious organisms, stage one operating time, time between resection and reimplantation, chronic antibiotic suppression, and most serious infection-related procedure prior to the index resection are provided in Table 4 for successfully treated cases based on spacer type and the use of IM dowels. Treatment success in the three spacer/IM dowel groups did not vary based on sex $(p=0.824)$, age $(p=0.456)$, or BMI $(p=0.111)$. There were 
no differences in the proportion of each group with antibiotic resistant organisms $(p=$

0.089). As would be expected, stage one operating time significantly increased commensurate with the amount of time required for the construction of spacers and dowels $(p=0.002)$. The time between resection and reimplantation did not differ based on spacer and dowel constructs $(p$

$194=0.822$ ). The proportion of patients on chronic antibiotic suppression following reimplantation did not statistically differ in the three spacer/IM dowel groups $(p=0.399)$.

Significantly fewer patients with static spacers and IM dowels (26.6\%), followed by patients with articulating spacers without dowels (59.1\%), and those with articulating spacers with dowels $(\mathbf{7 5 . 0 \%})$ had no infection-related procedures prior to the index resection $\left(\mathrm{X}^{2}=7.10, p=0.029\right)$.

\section{Discussion}

The use of evidence-based maximally effective treatment methods for PJI is second in urgency and importance only to the prevention and eradication of PJI itself. In the best case scenario, infected total and revision knees result in a devastating financial burden to patients and the healthcare system and exact a heavy emotional and functional toll on patients and their families. In the worst case scenario, devastating disability may ensue as a result of fusion or amputation. PJI is the antithesis of the functional independence and full, healthy lifestyle total joint arthroplasty surgeons strive to achieve for patients and therefore, appropriate and effective treatment strategies must be optimized to minimize the burden and maximize patient outcomes. The efficacy of articulating and static spacers in two-stage treatment for periprosthetic

210 knee infection has been studied [1-4] but there is a lack of data on the efficacy of IM dowels, a 211 potentially important tool to facilitate treatment and eradication of PJI of the knee via focused 212 delivery of antibiotics into the IM canals of the femur and tibia. Our examination of the 
effectiveness of IM dowels in a demographically typical resection sample supports existing studies demonstrating greater treatment success with articulating spacers compared to static spacers, although existing studies have not assessed whether IM dowels provide additional dowels do not improve upon the superior treatment efficacy provided by articulating compared to static spacers. Two stage treatment success rates were high when articulating spacers were used whether or not IM dowels were used, especially in the presence of chronically infected poor hosts with compromised extremities.

This study does have limitations. First it is retrospective in design and lacks the scientific rigor of randomization with respect to spacer type or presence of IM dowels. Second, due to the variability and complicated nature of PJI after TKA, we had to account for two variables: articulating versus static spacers with or without IM dowels. This effected the ability to isolate the variable of primary interest, the presence of high dose antibiotic IM dowels. Finally, there is a treatment bias that is evident with the surgeon decision to use a specific spacer type, in that significantly more patients with static spacers and IM dowels $(86.4 \%)$, followed by patients with articulating spacers and IM dowels (78.6\%), and articulating spacers without IM dowels (57.1\%) were chronically infected poor hosts with compromised extremities $(p=0.033)$. Further, patients with static spacers had on average a greater number of infection related surgical procedures than patients with articulating spacers $(p=0.029)$, suggesting an increased level

\section{2 of complexity and potential worse prognosis for infection eradication in those patients with}

233 prior infection procedures. Despite these limitations, this study does have noteworthy

234 strengths. First, this study includes a relatively large sample size, which is difficult to achieve in 235 this particular topic and treatment modality. Second, the variability of spacer type and presence 
of IM dowels was accounted for statistically to isolate each variable and correlation with treatment success.

Our study provides value in so far as, to the best of our knowledge, it is the only

239 investigation to date quantifying the effectiveness of IM dowels in the treatment of periprosthetic

240 knee infection. Further, the commonly quoted incidence of approximately $40 \%$ of

241 intramedullary canals having positive cultures at the time of resection currently lacks clinical

242 validation and the findings reported here provide corroboration via clinical outcome data. We

243 encourage additional research to verify our observation that the use of IM dowels did not

244 enhance infection eradication above and beyond that observed for articulating spacers alone,

245 including in the worst cases involving chronically infected poor hosts with compromised 246 extremities. 
1. Voleti PB, Baldwin KD, Lee GC. Use of static or articulating spacers for infection following total knee arthroplasty: a systematic literature review. The Journal of bone and joint surgery American volume 95(17): 1594, 2013

2. Pivec R, Naziri Q, Issa K, Banerjee S, Mont MA. Systematic review comparing static and articulating spacers used for revision of infected total knee arthroplasty. The Journal of arthroplasty 29(3): 553, 2014

3. Ding H, Yao J, Chang W, Liu F. Comparison of the efficacy of static versus articular spacers in two-stage revision surgery for the treatment of infection following total knee arthroplasty: a meta-analysis. Journal of orthopaedic surgery and research 12(1): 151, 2017

4. Guild GN, 3rd, Wu B, Scuderi GR. Articulating vs. Static antibiotic impregnated spacers in revision total knee arthroplasty for sepsis. A systematic review. The Journal of arthroplasty 29(3): 558, 2014

5. Abdel M, Barreira P, Battenberg A, Berry D, Blevins K, Font-Vizcarra L, Frommelt L, Goswami K, Greiner J, Janz V, Kendoff DO, Limberg AK, Manrique J, Moretti B, Murylev V,

266 Knee Section, Treatment, Two-Stage Exchange Spacer-Related: Proceedings of International

267 Consensus on Orthopedic Infections. The Journal of arthroplasty, 2018

268 6. Hanssen AD, Spangehl MJ. Practical applications of antibiotic-loaded bone cement for 269 treatment of infected joint replacements. Clinical Orthopaedics and Related Research (427): 79, 
271 7. McPherson EJ, Patzakis MJ, Gross JE, Holtom PD, Song M, Dorr LD. Infected total knee

272 arthroplasty. Two-stage reimplantation with a gastrocnemius rotational flap. Clinical

273 Orthopaedics and Related Research (341): 73, 1997

274 8. McPherson EJ, Tontz W, Jr., Patzakis M, Woodsome C, Holtom P, Norris L, Shufelt C.

275 Outcome of infected total knee utilizing a staging system for prosthetic joint infection. American

276 journal of orthopedics (Belle Mead, NJ) 28(3): 161, 1999

277 9. Parvizi J, Gehrke T. Definition of periprosthetic joint infection. The Journal of arthroplasty

278 29(7): 1331, 2014

279 


\section{Acknowledgements}

The project described was supported by the Indiana University Health - Indiana School of Medicine Strategic Research Initiative. 


\section{Figure Legends}

Figure 1. Radiographic images of spacer and dowel constructs. (A) Pre-fabricated articulating spacer with Steinmann pin IM dowels, (B) Molded antibiotic tibial and femoral spacers without IM dowels, (C) Molded cement femoral components and all-poly tibial inserts implanted with high-dose antibiotic cement without IM dowels, and (D) Static spacer with cement gun nozzle IM Dowels.

Figure 2. Overall treatment success rates (left) and success rates in chronically infected poor hosts (right) based on spacer type and the use of IM dowels. 


\begin{tabular}{|l|}
\hline Table 2: McPherson et al.'s Classification System for PJI \\
\hline Infection Type \\
\hline I. Early postoperative ( $\leq 4$ weeks postoperative) \\
\hline II. Acute hematogenous infection ( $\leq 4$ weeks duration) \\
\hline III. Late chronic infection (> 4 weeks duration) \\
\hline Systemic Host Grade (Medical/Immune Status) \\
\hline A. Uncompromised (no compromising factors) \\
\hline B. Compromised ( $\leq$ compromising factors) \\
\hline C. Significant compromise (> 2 compromising factors) \\
\hline Local Extremity Grade \\
\hline 1. Uncompromised (no compromising factors) \\
\hline 2. Compromised ( $\leq$ compromising factors) \\
\hline 3. Significant compromise (> 2 compromising factors) \\
\hline
\end{tabular}


Table 1: Distribution of Femoral and Tibial Dowels Based on Spacer Type and Method of Dowel Construction

\begin{tabular}{|l|c|c|c|c|}
\hline & \multicolumn{3}{|c|}{ Dowel Type } & \\
\hline & $\begin{array}{c}\text { Steinmann } \\
\text { Pin }\end{array}$ & $\begin{array}{c}\text { Reconstruction } \\
\text { Nail }\end{array}$ & $\begin{array}{c}\text { Hardened } \\
\text { Cement }\end{array}$ & Total \\
\hline Static Spacers With Dowels & & & & \\
\hline Femur \& Tibia & 13 & 3 & 6 & 22 \\
\hline Articulating Spacer with Dowels & & & & \\
\hline Femur \& Tibia & 9 & 0 & 1 & 10 \\
\hline Femur Only & 0 & 0 & 3 & 3 \\
\hline Tibia Only & 1 & 0 & 0 & 1 \\
\hline
\end{tabular}


Table 3: Prosthetic Joint Infection Classification Based on Spacer Type and the Use of IM Dowels

\begin{tabular}{|l|c|c|c|}
\hline N & $\begin{array}{c}\text { Static spacer with IM } \\
\text { dowels }\end{array}$ & $\begin{array}{c}\text { Articulating spacer with } \\
\text { IM dowels }\end{array}$ & $\begin{array}{c}\text { Articulating spacer } \\
\text { without IM dowels }\end{array}$ \\
\hline Stage & 22 & 14 & 49 \\
\hline I-A-1 to I-C-3 & 0.0 & & $\mathbf{2 4 . 5}$ \\
\hline II-A-1 to II-C-3 & $\mathbf{4 . 6}$ & $\mathbf{1 4 . 3}$ & $\mathbf{1 8 . 4}$ \\
\hline III-A-1 to III-A-3 & $\mathbf{4 . 6}$ & $\mathbf{7 . 1}$ & 0.0 \\
\hline III-B-1 & 0.0 & 0.0 & 0.0 \\
\hline III-B-2 & $\mathbf{1 3 . 6}$ & 0.0 & $\mathbf{2 4 . 5}$ \\
\hline III-B-3 & $\mathbf{2 2 . 7}$ & $\mathbf{1 4 . 3}$ & $\mathbf{1 0 . 2}$ \\
\hline III-C-1 & $\mathbf{4 . 6}$ & $\mathbf{7 . 1}$ & 0.0 \\
\hline III-C-2 & $\mathbf{1 3 . 6}$ & 0.0 & $\mathbf{1 0 . 2}$ \\
\hline III-C-3 & $\mathbf{3 6 . 4}$ & $\mathbf{5 0 . 0}$ & $\mathbf{1 2 . 2}$ \\
\hline
\end{tabular}

Gray shading demarcates stages associated with chronically infected poor hosts with compromised extremities 
Table 4: Characteristics of Successfully Treated Cases Based on Spacer Type and the Use of IM Dowels

\begin{tabular}{|c|c|c|c|c|c|}
\hline & 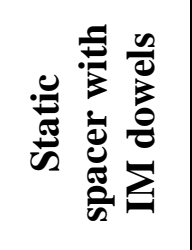 & 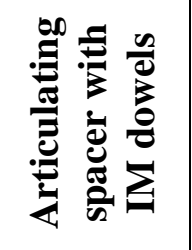 & 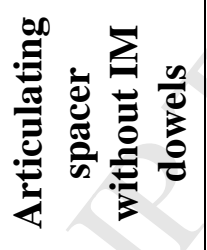 & Statistic & $p$ \\
\hline $\mathrm{N}$ & 15 & 12 & 44 & & \\
\hline$\%$ Female & 46.7 & 58.3 & 50.0 & $X^{2}=0.386$ & 0.824 \\
\hline Mean (SD) Age in Years & $64.7(9.6)$ & $69.0(8.8)$ & $65.4(9.0)$ & $\mathrm{F}=0.79$ & 0.456 \\
\hline Mean (SD) BMI in $\mathrm{kg} / \mathrm{m}^{2}$ & $39.5(9.6)$ & $37.3(6.3)$ & $34.1(9.3)$ & $\mathrm{F}=2.28$ & 0.111 \\
\hline \multicolumn{6}{|l|}{$\%$ Infectious Organism } \\
\hline NGTD & 33.2 & 8.3 & 36.4 & $*$ & $*$ \\
\hline CoNS & 13.3 & 41.7 & 15.9 & & \\
\hline MRSA & 6.7 & 16.8 & 0.0 & & \\
\hline MSSA & 6.7 & 8.3 & 25.0 & & \\
\hline Streptococcus & 6.7 & 8.3 & 9.1 & & \\
\hline Other & 6.7 & 8.3 & 6.8 & & \\
\hline Polymicrobial excluding Resistant Organisms & 20.0 & 0.0 & 4.5 & & \\
\hline Polymicrobial including Resistant Organisms & 6.7 & 8.3 & 2.3 & & \\
\hline$\%$ Antibiotic Resistant Organism $=$ Yes & 20.0 & 27.3 & 3.6 & $X^{2}=4.832$ & 0.089 \\
\hline Mean (SD) Stage One Operating Time in Minutes & $\begin{array}{l}137.4 \\
(23.8)\end{array}$ & $\begin{array}{l}117.8 \\
(33.9) \\
\end{array}$ & $\begin{array}{l}106.6 \\
(26.7) \\
\end{array}$ & $F=7.14$ & 0.002 \\
\hline $\begin{array}{l}\text { Mean (SD) Time Between Resection and } \\
\text { Reimplantation in Months }\end{array}$ & $2.8(1.4)$ & $2.7(1.0)$ & $2.6(0.8)$ & $F=0.20$ & 0.822 \\
\hline $\begin{array}{l}\text { \% Chronic Antibiotic Suppression Following } \\
\text { Reimplantation }\end{array}$ & 21.4 & 20.0 & 8.6 & $X^{2}=1.837$ & 0.399 \\
\hline \multicolumn{6}{|l|}{$\begin{array}{l}\text { \% Most Serious Infection-Related Procedures Prior } \\
\text { to Index Resection }\end{array}$} \\
\hline Treatment of TKA Arthrotomy Wound & 6.7 & 0.0 & 4.6 & $*$ & $*$ \\
\hline Arthroscopic I\&D & 0.0 & 0.0 & 4.6 & & \\
\hline Open I\&D without Liner Exchange & 6.7 & 8.3 & 15.9 & & \\
\hline Open I\&D with Liner Exchange & 6.7 & 0.0 & 9.0 & & \\
\hline Resection and Reimplantation at OSH & 53.3 & 16.7 & 6.8 & & \\
\hline None & 26.6 & 75.0 & 59.1 & & \\
\hline
\end{tabular}


*Chi-Square invalid due to low cell counts

NGTD $=$ no growth to date

CoNS = coagulase-negative staphylococci

MRSA = Methicillin-resistant Staphylococcus aureus

MSSA = Methicillin-sensitive Staphylococcus aureus

Other $=$ Corynebacterium, Enterococcus, Pasturella multocida, Pseudomonas aeruginosa

Polymicrobial $=$ Combinations of CoNS, Corynebacterium species, Enterococcus, Escherichia coli, Group B streptococcus, Group C streptococcus, Kebsiella oxytoca, MRSA, MSSA, Peptostreptococcus, Providencia, Prevotella anaerobic gram-negative rods, Pseudomonas, Staphylococcus aureus, Staphylococcus lugdunensis, Vancomycin-resistant Enterococcus

$\mathrm{I} \& \mathrm{D}=$ irrigation and debridement

$\mathrm{OSH}=$ outside hospital 
$\square$ Articulating Spacers without Dowels

$\square$ Articulating Spacers with Dowels

$\square$ Static Spacers with Dowels

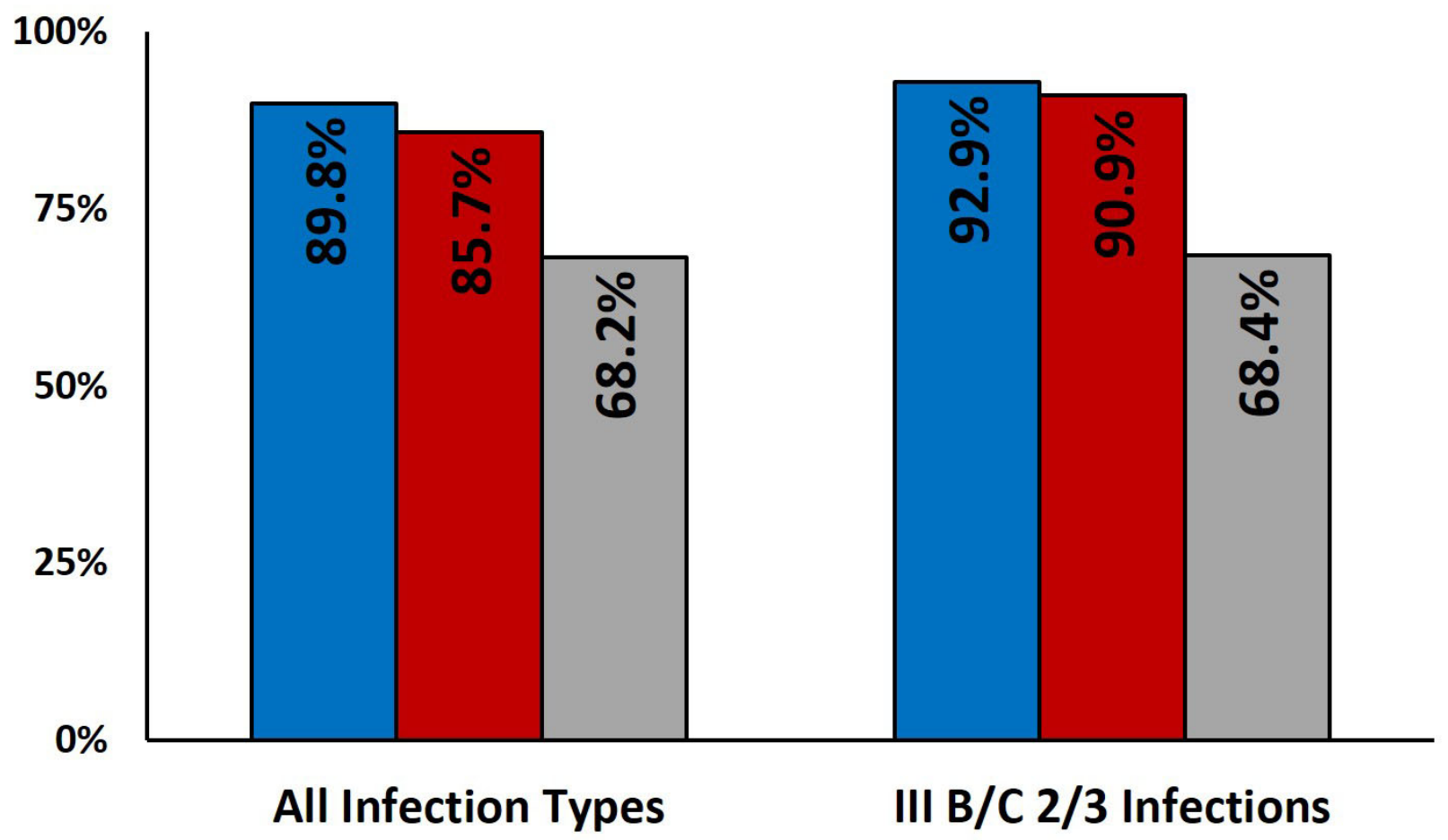



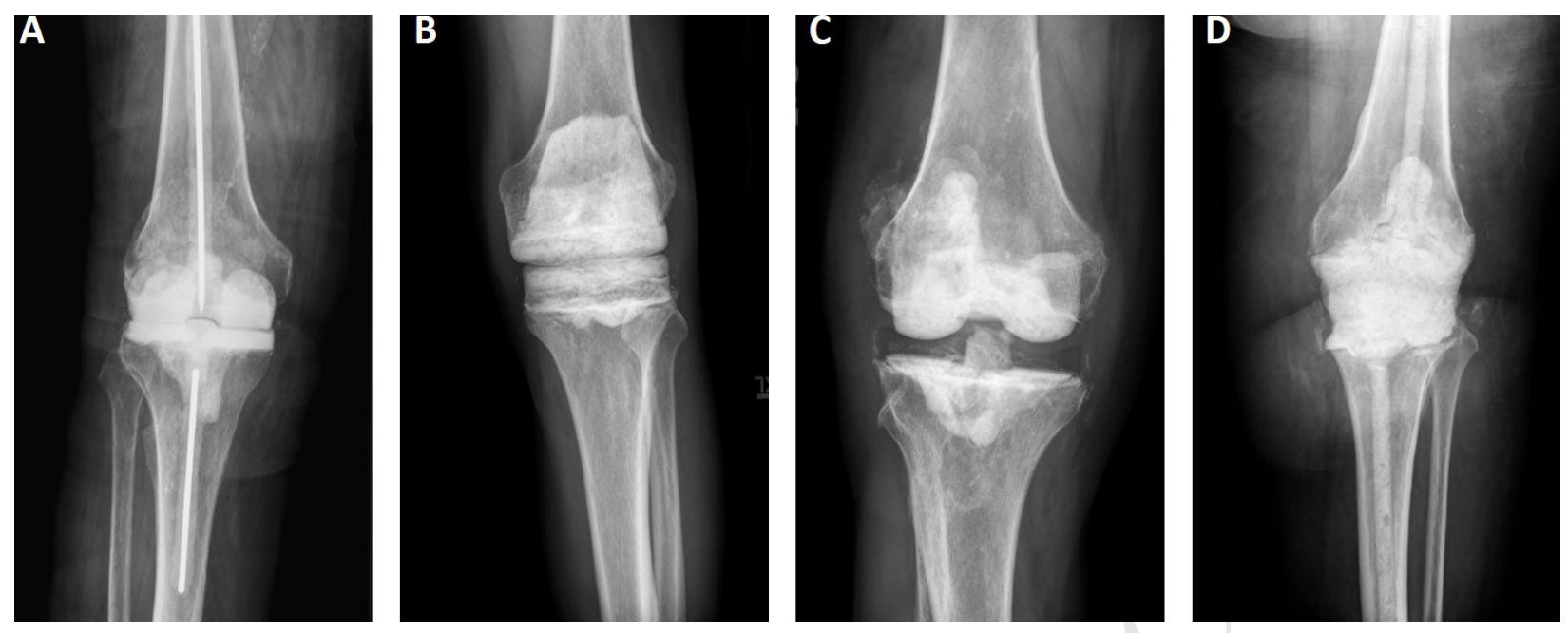